\title{
Fault Detection for Network Control System with Multiple Packet Loss
}

\author{
Yuqing $\mathrm{Li}^{1}$, Qun Zhang ${ }^{2}$ \\ ${ }^{1}$ China University of Geosciences, Wuhan430074, China; \\ ${ }^{2}$ China University of Geosciences, Wuhan430074, China; \\ 328463200@qq.com
}

Keywords: Networked control, Data packet dropout, Fault diagnosis

\begin{abstract}
The fault diagnosis problems for Networked control systems with multiple packet loss is studied. Residual model is designed to meet the minimum system implemented. By choosing the appropriate Lyapunov function, the sufficient conditions for the robust stochastic stability of the filter fault diagnosis for the controller designed by the proposed model are demonstrated by Lyapunov stability theory and linear matrix inequality theory. The simulation example shows the effectiveness of the method.
\end{abstract}

\section{Introduction}

Networked Control System (NCS) is a closed-loop control system connected by a real-time network. The network based control system has the advantages of less connection, low cost, resource sharing, easy to install, maintain, extend and fault diagnosis. But the intervention of communication network, limited by bandwidth and transmission is not reliable, the network delay, data packet loss is inevitable, once the system fails, the loss will be difficult to measure. So NCS fault detection is very important.

In this paper, we consider the problem of fault diagnosis for networked control systems with multiple data packet dropout. The residual model of the system is designed.

\section{Problem description}

The discrete model of the NCSs system is assumed to exist as the packet loss is as follows:

$$
\left\{\begin{array}{c}
x(k+1)=A x(k)+B u(k)+E w(k)+G f(k), \\
y(k)=\Theta(k) C x(k)+D u(k)+F w(k)+H f(k)
\end{array}\right.
$$

$\Theta(k) \stackrel{\Delta}{=} \operatorname{diag}\left\{\theta_{1}(k), \theta_{2}(k), \cdots, \theta_{q}(k)\right\}$ expression more than a collection of data packet loss, $x(k), u(k), y(k), w(k), f(k)$ respectively show that the state vector ,the known input vector, the output vector of the discrete model and discretization of the unknown inpuvector and fault measurement vector.

The assumption is that the norm is bounded. A, B, C, D, E, F, G, H are not completely known.There is a convex polyhedron model in the following form:

$$
\begin{aligned}
& \Omega(a)=(A, B, C, D, E, F, G, H) \in \Psi \\
& \Psi=\left\{\Omega(a) \mid \Omega(a)=\sum_{i=1}^{N} a_{i} \Omega_{i} ; \sum_{i=1}^{N} a_{i}=1, a_{i} \geq 0\right\}, \text { said the first a convex vertex } \\
& \Omega_{i}=\left(A_{i}, B_{i}, \cdots, H_{i}\right)
\end{aligned}
$$

Fault diagnosis filter hypothesis is as follows: $\left\{\begin{array}{c}\hat{x}(k+1)=A_{F} x(k)+B_{F} y(k) \\ r(k)=C_{F} \hat{x}(k)\end{array}\right.$

$\hat{x} \in R^{n}, r(k) \in R^{s}$ respectively design the fault diagnosis of filter state vector and residual vector. $A_{F}, B_{F}, C_{F}$, are pending matrix, $\hat{f}(k)=W(k) f(k)$ is the given stable matrix.

Assumes $\hat{f}(k)=W(k) f(k)$ exist the minimal implementation, and has the following form: 


$$
\begin{gathered}
\left\{\begin{array}{l}
- \\
x(k+1)=A_{w} x(k)+B_{w} f(k) \\
\hat{f}(k)=C_{w} x(k)+D_{w} f(k)
\end{array}\right. \\
\xi(k)=\left[\begin{array}{lll}
x^{T}(k) & x^{T} & -\overline{x^{T}}(k)
\end{array}\right]^{T}, d(k)=\left[\begin{array}{lll}
u^{T}(k) & w^{T}(k) & f^{T}(k)
\end{array}\right]^{T}
\end{gathered}
$$

$e(k) \stackrel{\Delta}{=} r(k)-\hat{f}(k), \stackrel{-\Delta}{\Theta}=\operatorname{diag}\left\{u_{1}, u_{2}, \cdots, u_{q}\right\}, \tilde{\Theta}(k) \stackrel{\Delta}{=} \Theta(k)-\stackrel{-}{\Theta}$ So the residual error model of system is described below: $\left\{\begin{array}{c}\xi(k+1)=\bar{A}(a, k) \xi(k)+\bar{B}(a) d(k) \\ e(k)=\bar{C} \xi(k)+\bar{D} d(k)\end{array}\right.$

$$
\begin{aligned}
& \text { In the above formula, } \bar{A}(a, k)=\bar{A}(a)+\left[\begin{array}{ccc}
0 & 0 & 0 \\
B_{F} \tilde{\Theta}(k) C(a) & 0 & 0 \\
0 & 0 & 0
\end{array}\right], \bar{B}(a)=\left[\begin{array}{ccc}
B(a) & E(a) & G(a) \\
B_{F} D(a) & B_{F} F(a) & B_{F} H(a) \\
0 & 0 & B_{w}
\end{array}\right] \text {, } \\
& \bar{A}(a)=\left[\begin{array}{ccc}
A(a) & 0 & 0 \\
B_{F} \bar{\Theta} C(a) & A_{F} & 0 \\
0 & 0 & 0
\end{array}\right], \quad \bar{C}=\left[\begin{array}{lll}
0 & C_{F} & -C_{w}
\end{array}\right] \bar{D}=\left[\begin{array}{lll}
0 & 0 & -D_{w}
\end{array}\right]
\end{aligned}
$$

From formula (1) - (4) analysis shows that on multiple data packet dropout of NCSs fault diagnosis problem can be summarized as: given positive number $\gamma$, appropriate design of fault detection filter (2) of undetermined matrix $A_{F}, B_{F}, C_{F}$, residual error model of the system (4) robust stochastically stable. And meet the following conditions at zero initial conditions:

$$
\left(E\left\{\sum_{k=0}^{\infty} e^{T}(k) e(k)\right\}\right)^{\frac{1}{2}}<\gamma\|d\|_{2}
$$

Corresponding residual evaluation function $J(r)$ and the threshold $J_{t h}$ is defined as $J(r)=\sqrt{\sum_{l=0}^{L} r^{T}(l) r(l)}, \quad \mathrm{L}$ is on behalf of the evaluation time of step. Fault diagnosis system can be $J_{t h}=\sup _{u \in l_{2}, w l_{2}, f=0} E\{J(r)\}$

obtained based on the following judgment: $\left\{\begin{array}{l}J(r) \leq J_{t h} \rightarrow \text { 正常 } \\ J(r)>J_{t h} \rightarrow \text { 故障 }\end{array}\right.$

The following theorem shows the residual model of the system (4) robust stochastic stable and sufficient condition to meet the $\left(E\left\{\sum_{k=0}^{\infty} e^{T}(k) e(k)\right\}\right)^{\frac{1}{2}}<\gamma\|d\|_{2}$ is established at zero initial conditions.

\section{Main results}

Theorem 1 Given positive number $\gamma$, design suitable pending matrix $A_{F} B_{F}, C_{F}$, if the matrix $P(a)$ exists, and satisfies $\begin{aligned} & P(a)>0 \text {, to make the following inequality holds: } \\ & X(a),\end{aligned}$

$$
\begin{aligned}
& {\left[\begin{array}{ccccc}
-P(a) & 0 & \overline{A^{T}}(a) X^{T}(a) & \Phi^{T}(a) & \bar{C}^{T} \\
0 & -\gamma^{2} I & \overline{B^{T}}(a) X^{T}(a) & 0 & \bar{D}^{T} \\
X(a) \bar{A}(a) & X(a) \bar{B}(a) & P(a)-X(a)-X^{T}(a) & 0 & 0 \\
\Phi(a) & 0 & 0 & \mathrm{Y}(a) & 0 \\
\bar{C} & \bar{C} & 0 & 0 & -I
\end{array}\right]<0 \quad \Phi(a)=\left[\begin{array}{lll}
\Gamma_{1}^{T}(a) X_{22}^{T}(a) & \cdots & \Gamma_{q}^{T}(a) X_{22}^{T}(a)
\end{array}\right]^{T}, \Gamma_{l}(a)=\left[\begin{array}{lll}
B_{F} \Sigma_{l} C(a) & 0 & 0
\end{array}\right]} \\
& \sum_{l}=\operatorname{diag}\{\underbrace{0, \cdots, 0, \sigma_{i}}_{l-1}, \underbrace{0, \cdots, 0}_{a-l}\}, l=1,2, \cdots, q Y(a)=\operatorname{diag}\{\underbrace{\Lambda(a), \cdots, \Lambda(a)}_{q}\}, \Lambda(a)=P_{22}(a)-X_{22}(a)-X^{T}{ }_{22}(a)
\end{aligned}
$$

Then have the conclusions: the residual model of the system (4) establishment of robust stochastic stability and satisfies the formula (5) at zero initial conditions.

In order to determine the pending matrix $A_{F}, B_{F}, C_{F}$, in the fault diagnosis of filter, the following theorems is given. 
Theorem 2 For the given constant $\varepsilon$ and $\gamma>0$, there exist the following matrix $P_{i}=\left[\begin{array}{ccc}P_{11 i} & P_{12 i} & P_{13 i} \\ P_{12 i}^{T} & P_{22 i} & P_{23 i} \\ P_{13 i}^{T} & P_{23 i}^{T} & P_{33 i}\end{array}\right]>0, X_{i}=\left[\begin{array}{ccc}X_{11 i} & \varepsilon X_{22} & X_{13 i} \\ X_{21 i} & X_{22} & X_{23 i} \\ X_{31 i} & 0 & X_{33 i}\end{array}\right], \hat{A_{F}}, \hat{B_{F}}, \hat{C_{F}}$, which can meet the linear $\operatorname{matrix}\left\{\begin{array}{c}\Xi_{i i}<0, i=1,2, \cdots, N \\ \Xi_{i j}+\Xi_{j i}<0,1 \leq i<j \leq N\end{array}\right.$ inequality $\cdot \Xi_{i j}=\left[\begin{array}{ccccc}-P_{1} & 0 & M_{i j}^{T} & \Psi_{i j}^{T} & \hat{C^{T}} \\ 0 & -\gamma^{2} I & N_{i j}^{T} & 0 & D^{T} \\ M_{i j} & N_{i j} & P_{i}-X_{i}-X_{i}^{T} & 0 & 0 \\ \Psi_{i j} & 0 & 0 & Y_{i} & 0 \\ \hat{C} & \bar{D} & 0 & 0 & -I\end{array}\right]$

On the premise of zero initial conditions meet the $\left(E\left\{\sum_{k=0}^{\infty} e^{T}(k) e(k)\right\}\right)^{\frac{1}{2}}<\gamma\|d\|_{2}$ was established.

\section{Simulation Example}

A case study of the fault diagnosis of a high pressure boiler reactor with a mixed type reactor was chosen to assume that the system has two data packet $\theta_{1}(k), \theta_{2}(k)$ lost:

$$
\left\{\begin{array}{c}
x(k+1)=\left[\begin{array}{cc}
0.9825 & -0.0014 \\
-0.0273 & 0.9128
\end{array}\right] x(k)+\left[\begin{array}{cc}
-0.0798+\rho & 0.0256 \\
0.0805+\rho & 0.4052
\end{array}\right] u(k) \\
y(k)=\left[\begin{array}{ll}
1 & 0 \\
0 & 1
\end{array}\right] x(k)+\left[\begin{array}{c}
0 \\
0.1
\end{array}\right] w(k)
\end{array}\right.
$$

The fault matrix is $G=\left[\begin{array}{c}-0.0798+\rho \\ 0.0805+\rho\end{array}\right]$. Matrix $A_{w}=0.1, B_{w}=0.25, C_{w}=0.5, D_{w}=0$

If the given $\gamma=0.2, \varepsilon=0.01$, fault diagnosis filter gain is obtained as follows:

Select the threshold $J_{t h}=\sup _{u \in l_{2}, w \in l_{2}, f=0} E\{J(r)\} \sup _{u \in l_{2}, w \in l_{2}, f=0} E\left\{\sqrt{\sum_{l=0}^{L} r^{T}(l) r(l)}\right\}=0.0058$

MATLAB simulation software of the hybrid reactor model system of the high pressure boiler, the system of the residual response and residual evaluation function is obtained:

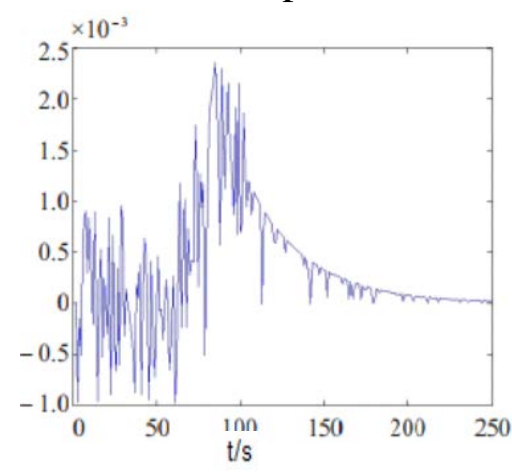

Fig. 1 System residual response

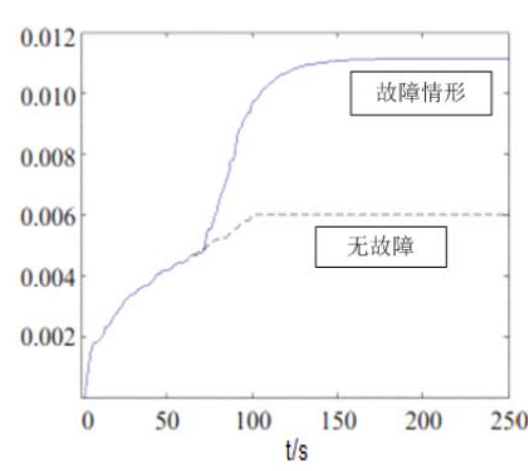

Fig. 2 System residual evaluation function

By MATLAB simulation results of the system residuals corresponding figure 2 and the evaluation function can also be seen in the comparison of 1 : in the sampling time between $k=70 s \rightarrow 90 s$, the system of two data packet loss response is more obvious change, the corresponding residual evaluation function in the failure curve is obvious, and the evaluation function is stable. 


\section{Summary}

In this paper, the NCSs problem of multi data packet dropout is modeled and analyzed, and the corresponding fault diagnosis filter is designed, and the minimal realization and system residual model are given.By constructing Lyapunov function and linear matrix inequality technique, the sufficient condition of robust stochastic stability is demonstrated, and the calculation method of undetermined matrix vector in the stability analysis is presented.The effectiveness of the method is verified by example simulation.

\section{References}

[1] Huo Z, Fang H. Fault-tolerant Control Research on Networked Control System with Random Time-delays[J]. Information and Control,2006,35(5):584-587.

[2] Huo Z, Fang H. Research on Robust Fault Detection for NCS with Long Delay Based on LMI[J]. Advances in Systems Science and Application,2005,5(3):404-410.

[3] Ge-luo Suo ,Xian-hui Yang .Research on system stability for Networked Control System with transmission delay and packet loss [J]. Control and Decision,2006, 21(2):201-204.

[4] Mehrdad, Sahebsaraa, Tongwen Chenb. Optimal $H_{\infty}$ filtering in networked control systems with multiple packet dropouts[J]. Systems and Control Letters, 2008, 57(1):696-702.

[5]Jian-dong Sun ,Jing-ping Jiang .Stability of uncertain networked control systema [J].Procedia Engineering,2011,24:551-557.

[6] Zhang P, Ding E, Frank P M. Study on detecting multiplicative faults in linear dynamic systems [C]. Proceedings of the American Control Conference, 2003: 1776-1781.

[7]Wang Z, Huang L, Yang X. Hळ performance for a class of uncertain stochastic nonlinear Markovian jump systems with time-varying delay adaptive control method[J]. Applied Mathematical Modelling.2011,35(4):1983-1993. 